\title{
On the Hankel Singular Values of Input Time Delay Systems
}

\author{
I Cheol HwAnG*, Hirohisa Nishino* and Yoshito OHTA*
}

\begin{abstract}
This paper derives a method to calculate the Hankel singular values of input time delay systems. The method uses the property that the gramian is an integral operator with a semi-seperable kernel function, and hence is represented as a Volterra operator plus a finite-dimensional operator. The Hankel singular values are obtained by calculating a transcendental equation involving the determinant of the associated finite-dimensional matrix functions. The relation between this result and the result in the literature is explained explicitly, by coordinate transformation. Futhermore, lower and upper bounds on their values are estabilished using the minimax properties of the eigenvalues for a compact and non-negative self-adjoint operator. This result shows that as the length of delay increases, all the Hankel singular values have a monotone increasing property.
\end{abstract}

Key Words : input time delay systems, gramian operator, Hankel singular value, semi-seperable kernel function, minimax theorem

\section{Introduction}

In control theory, particularly, in case of solving $\mathscr{H}^{\infty}$ control or model reduction problem, it is well known that Hankel singular values play an important role by a number of researches. Glover first considered the Hankel singular values in the $\mathscr{L}_{\infty}$ approximation problem of finite dimensional systems ${ }^{1)}$. Since then, the Hankel singular values were also studied in the $\mathscr{L}_{\infty}$ approximation problem of infinitedimensional systems because of their usefulness in the design of robust finite dimensional controllers ${ }^{2), 3)}$.

The Hankel singular values of systems with point delay in control were first obtained in solving the $\mathfrak{H}^{\infty}$ sensitivity optimization problem ${ }^{4), 5}$. In particular, Zhou and Khargonekar ${ }^{5)}$ derived an analytic method to compute the Hankel singular values by solving a transcendental equation involving the exponential of a Hamiltonian matrix. In a similiar way, Glover et al. ${ }^{6)}$ computed their values of input time delay systems and considered their asymptotic behaviours. Recently, Kojima and Ishijima ${ }^{7)}$ computed the Hankel

$\dagger$ Presented 33rd SICE Annual Conference(1994•7), 37th Japan Joint Automatic Control Conference (1994•11)

* Faculty of Engineering, Osaka University, 2-1,

Yamadaoka, Suita

(Received January 4, 1995) singular values using the gramian.

From the above results ${ }^{4)-7)}$, we have a question why the Hankel singular values for input time delay systems with infinite-dimensional can be obtained by calculating the determinent of a finite-dimensional matrix functions. We focus on the property of the gramian, and show that it is an integral operator whose kernel has a structure called semi-seperable ${ }^{8)}$. Indeed, it is this property that reduces the computation of infinite dimensional eigenvalue problem a transcendental equation. Futhermore, using the minimax properties of the eigenvalues for a compact and non-negative self-adjoint operator, we give upper and lower bounds on the Hankel singular values. As a corollary of this result, we show the fact that as the length of delay increases, all the Hankel singular values have a monotone increasing property.

Section 2 describes system with point delay in the control as a difference equation using the lifting technique $^{9)}$. In section 3 , the gramians are given and their properties are studied. Section 4 derives a method to compute the Hankel singular values and shown the relation between the result of this paper and the previous results ${ }^{4)-7)}$. In section 5 , the minimax theorem for compact and nonnegative self-adjoint operators is applied to give upper and lower bounds 
of the Hankel singular values. As a corollary, it is proved that as the length of delay increases, all the Hankel singular values have a monotone increasing property. And these results are explained through a simple example in section 6 .

\section{Basic Model and Notations}

We consider an input time delay system given by $G(s)=\exp (-s L) F(s)$, where $L>0$ is the length of time delay and $F(s)$ is a stable and strictly proper rational transfer function. A differential-difference equation of $G(s)$ is given by

$$
\begin{aligned}
& \dot{x}(t)=A x(t)+B u(t-L), \\
& y(t)=C x(t),
\end{aligned}
$$

where $x(t) \in \mathscr{R}^{n}, u(t) \in \mathscr{R}^{m}$, and $y(t) \in \mathscr{R}^{p}$ are state, control input and observable output variables, respectively. $\mathscr{R}^{i}$ denotes $i$-dimensional real Euclidean space. $[A, B, C]$ is an $n$-dimensional minimal realization of $F(s)$. For $G(s)$, we assume that the pair ( $A$, $B)$ is controllable and the pair $(C, A)$ is observable. Using the lifting technique ${ }^{9)}$, the discrete-time system $\widehat{G}$ corresponding to the continuous-time system (2.1) can be defined by

$$
\begin{aligned}
& x_{i+1}=\widehat{A} x_{i}+\widehat{B} u_{i-1}, \\
& y_{i}=\widehat{C} x_{i}+\widehat{D} u_{i-1},
\end{aligned}
$$

where $x_{i} \cong x(i L) \in \mathscr{R}^{n}, u_{i} \cong u(i L+t) \in \mathscr{L}_{2}[0, L]$, and $y_{i} \cong y(i L+t) \in \mathscr{L}_{2}[0, L], i=0,1,2, \cdots, t \in[0, L] . \mathscr{L}_{2}[0$, $L]$ denotes the Hilbert space of square integrable functions on $t \in[0, L] . \quad \widehat{A}: \mathscr{R}^{n} \rightarrow \mathscr{R}^{n}, \hat{B}: \mathscr{L}_{2}[0, L] \rightarrow$ $\mathscr{R}^{n}, \hat{C}: \mathscr{R}^{n} \rightarrow \mathscr{L}_{2}[0, L]$, and $\hat{D}: \mathscr{L}_{2}[0, L] \rightarrow \mathscr{L}_{2}[0, L]$ are defined by

$$
\begin{aligned}
& \widehat{A} x_{i}=e^{A L} x_{i}, \quad \widehat{B} u_{i-1}=\int_{0}^{L} e^{A(L-s)} B u_{i-1}(s) d s, \\
& \widehat{C} x_{i}=C e^{A t} x_{i}, \hat{D} u_{i-1}=C \int_{0}^{t} e^{A(t-s)} B u_{i-1}(s) d s .
\end{aligned}
$$

Define $\xi_{i} \cong\left[x_{i} u_{i-1}\right]^{\prime} \in \mathscr{R}^{n} \times \mathscr{L}_{2}[0, L]$. Then $\xi_{i}, u_{i}$ and $y_{i}$ satisfy the following difference equation $\tilde{G}$ :

$$
\begin{aligned}
& \xi_{i+1}=\widetilde{A} \xi_{i}+\widetilde{B} u_{i}, \\
& y_{i}=\widetilde{C} \xi_{i},
\end{aligned}
$$

where $\tilde{A}: \mathscr{R}^{n} \times \mathscr{L}_{2}[0, L] \rightarrow \mathscr{R}^{n} \times \mathscr{L}_{2}[0, L], \tilde{B}: \mathscr{L}_{2}[0$, $L] \rightarrow \mathscr{R}^{n} \times \mathscr{L}_{2}[0, L]$, and $\widetilde{C}: \mathscr{R}^{n} \times \mathscr{L}_{2}[0, L] \rightarrow \mathscr{L}_{2}[0, L]$ are given by

$$
\tilde{A}:=\left[\begin{array}{cc}
\widehat{A} & \widehat{B} \\
0 & 0
\end{array}\right], \tilde{B}:=\left[\begin{array}{l}
0 \\
I
\end{array}\right], \tilde{C}:=\left[\begin{array}{cc}
\bar{C} & \bar{D}
\end{array}\right] .
$$

\section{Hankel and Gramian Operator}

An impulse response $h(t)$ of (2.1) is

$$
h(t)= \begin{cases}0 & , 0 \leq t \leq L \\ C \exp (A t) B, & L<t .\end{cases}
$$

Then the Hankel operator $\Gamma_{h}$ with impulse response $h(t)$ is defined by

$$
\begin{aligned}
\left(\Gamma_{h} f\right)(t) & =\int_{0}^{\infty} h(t+s) f(s) d s \\
& =\left\{\begin{array}{l}
\int_{L-t}^{\infty} C e^{A(t-L+s)} B f(s) d s, \quad 0 \leq t \leq L \\
\int_{0}^{\infty} C e^{A(t-L+s)} B f(s\} d s, \quad L<t
\end{array}\right.
\end{aligned}
$$

Note that since $h \in \mathscr{L}_{1}(0, \infty) \cap \mathscr{L}_{2}(0, \infty), \Gamma_{h}: \mathscr{L}_{2}(0$, $\infty) \rightarrow \mathscr{L}_{2}(0, \infty)$ is well-defined and a compact operator, which is bounded, with $\left\|\Gamma_{h}\right\| \leq|h(t)|_{1}{ }^{10)}$. Let $H_{G}$ : $l_{\mathscr{L}_{2}[0, L]}^{2} \rightarrow l_{\mathscr{L}_{2}[0, L]}^{2}$ be the Hankel matrix operator representation for the lifting system (2.4), where $l_{\mathscr{L}_{2}[0, L]}^{2}$ denotes the Hilbert space of square summable sequences composed with functions of $\mathscr{L}_{2}[0, L]$. The lifting for $\Gamma$ of (3.2) gives $H_{G}$, which is interpreted as the mapping from the past inputs, $u_{-i}$ to the future outputs, $y_{i}, i=0,1,2, \cdots$. that is,

$$
\left[\begin{array}{c}
y_{0} \\
y_{1} \\
\vdots
\end{array}\right]=\left[\begin{array}{ccc}
\tilde{C} \tilde{B} & \tilde{C} \tilde{A} \tilde{B} & \cdots \\
\tilde{C} \tilde{A} \tilde{B} & \cdots & \cdots \\
\vdots & \ddots & \ddots
\end{array}\right]\left[\begin{array}{c}
u_{-1} \\
u_{-2} \\
\vdots
\end{array}\right],
$$

Then, the controllability and observability gramians $\widetilde{P}, \widetilde{Q}$ of the system (2.4);

$$
\tilde{P}=\sum_{i=0}^{\infty} \tilde{A}^{k} \tilde{B} \tilde{B}^{*} \tilde{A}^{* k}, \tilde{Q}=\sum_{i=0}^{\infty} \tilde{A}^{* k} \tilde{C}^{*} \tilde{C} \tilde{A}^{k},
$$

are well defined on $\mathscr{R}^{n} \times \mathscr{L}_{2}[0, L]$ from the following theorem.

Theorem 1: Let $\tilde{P}$ and $\tilde{Q}$ be the controllability and the observability gramians defined by (3.4). Then

$$
\tilde{P}:=\left[\begin{array}{cc}
P & 0 \\
0 & I
\end{array}\right], \quad \tilde{Q}:=\left[\begin{array}{cc}
Q & K_{1}^{*} \\
K_{1} & K
\end{array}\right],
$$

are well-defined on $\mathscr{R}^{n} \times \mathscr{L}_{2}[0, L]$, where $P, Q$ are the positive definite Hermitian solutions to

$$
\begin{aligned}
& A P+P A^{*}+B B^{*}=0, \\
& Q A+A^{*} Q+C^{*} C=0 .
\end{aligned}
$$

And, for $f \in \mathscr{L}_{2}[0, L], z \in \mathscr{R}^{n}, K_{1}: \mathscr{R}^{n} \rightarrow \mathscr{L}_{2}[0, L]$, $K_{1}^{*}: \mathscr{L}_{2}[0, L] \rightarrow \mathscr{R}^{n}$, and $K: \mathscr{L}_{2}[0, L] \rightarrow \mathscr{L}_{2}[0, L]$ are defined by

$$
\left(K_{1} z\right)(t)=F_{1}(t) z, K_{1}^{*} f=\int_{0}^{L} G_{2}(s) f(s) d s,
$$




$$
\begin{aligned}
(K f)(t)= & F_{1}(t) \int_{0}^{t} G_{1}(s) f(s) d s \\
& +F_{2}(t) \int_{t}^{L} G_{2}(s) f(s) d s,
\end{aligned}
$$

where

$$
\begin{aligned}
& F_{1}(t)=B^{*} Q e^{A t}, G_{1}(t)=e^{-A t} B, \\
& F_{2}(t)=B^{*} e^{-A * t}, G_{2}(t)=e^{A * t} Q B .
\end{aligned}
$$

Proof: It is easily shown that $P, Q$ are the positive definite Hermitian solutions satisfying (3.6), (3.7) by

$$
\begin{aligned}
\sum_{i=0}^{\infty} \widehat{A}^{k} \widehat{B} \widehat{B}^{*} \widehat{A}^{* k} x & =\int_{0}^{\infty} e^{A t} B B^{*} e^{A * t} d t x \\
& =P x, \\
\sum_{i=0}^{\infty} \widehat{A}^{* k} \widehat{C}^{*} \widehat{C} \widehat{A}^{k} x & =\int_{0}^{\infty} e^{A * t} C^{*} C e^{A t} d t x \\
& =Q x .
\end{aligned}
$$

Thus a straightforward calculation of $\tilde{P}$ and $\tilde{Q}$ defined by (3.4) gives (3.5) and it is easily shown that they are well defined on $\mathscr{R}^{n} \times \mathscr{L}_{2}[0, L]$.

Note that $\tilde{Q}$ is compact, and the $(2,2)$ block is the integral operator on $t \in[0, L]$ with a semiseperable kernel function $k(t, s)^{8)}$;

$$
k(t, s)= \begin{cases}F_{1}(t) G_{1}(s), & 0 \leq s \leq t \leq L \\ F_{2}(t) G_{2}(s), & 0 \leq t<s \leq L .\end{cases}
$$

In order to examine the properties of $\tilde{P} \tilde{Q}$ we rewrite the operator $K$ of equation (3.9) as follows:

$$
K=H+K_{2} K_{1}^{*} \text {, }
$$

where $K_{1}^{*}: \mathscr{L}_{2}[0, L] \rightarrow \mathscr{R}^{n}$ is defined by (3.8) and $H$ : $\mathscr{L}_{2}[0, L] \rightarrow \mathscr{L}_{2}[0, L], K_{2}: \mathscr{R}^{n} \rightarrow \mathscr{L}_{2}[0, L]$ are defined by

$$
\begin{aligned}
(H f)(t)= & F_{1}(t) \int_{0}^{t} G_{1}(s) f(s) d s \\
& -F_{2}(t) \int_{0}^{t} G_{2}(s) f(s) d s, \\
\left(K_{2} z\right)(t)= & F_{2}(t) z .
\end{aligned}
$$

Then, $\tilde{P} \widetilde{Q}$ can be rewritten by

$$
\widetilde{P} \widetilde{Q}=\left[\begin{array}{cc}
0 & 0 \\
0 & H
\end{array}\right]+\left[\begin{array}{cc}
P Q & P K_{1}^{*} \\
K_{1} & K_{2} K_{1}^{*}
\end{array}\right],
$$

where the first term of the right hand side is a Volterra integral operator, the second term is finite rank.

With equation (3.11) we associate the following matrix :

$$
\tilde{S}(t):=\left[\begin{array}{rr}
G_{1}(t) F_{1}(t) & G_{1}(t) F_{2}(t) \\
-G_{2}(t) F_{1}(t) & -G_{2}(t) F_{2}(t)
\end{array}\right] .
$$

Note that $\widetilde{S}(t)$ is continuous function of time and is a square matrix of size $2 n \times 2 n$, where $2 n$ is the order of the representation (3.15).

The Hankel singular values, $\sigma(G)$, of input time delay systems can be given by the eigenvalues, $\lambda(\tilde{P} \tilde{Q})$, of $\tilde{P} \tilde{Q}$ due to the following lemma 1 of Curtain ${ }^{11)}$. lemma $1^{11)}$ : Suppose that $(\widetilde{C}, \widetilde{A}, \widetilde{B})$ is defined by (2.4). The non-zero singular values $\sigma_{i}, i=1,2, \cdots$, of its Hankel matrix operator, $H_{G}$ of (3.3), are in one-to-one correspondence with the eigenvalues $\sigma^{2}, i$ $=1,2, \cdots$, of $\tilde{P} \tilde{Q}$, where $\tilde{P}$ and $\tilde{Q}$ are the controllability and observability gramians given in (3.4), respectively.

\section{Hankel Singular Values}

Let define $\mu=\lambda^{-1}(\tilde{P} \tilde{Q})=\sigma^{-2}(G)$. From lemma 1, by examining the invertibility of $(I-\mu \tilde{P} \tilde{Q})$, we obtain the following theorem 2 .

Theorem 2: For each $\mu \in \mathscr{R}$, let $U(t ; \mu)$ be the unique $2 n \times 2 n$ matrix function determined by

$$
U(t ; \mu)=I_{2 n}+\mu \int_{0}^{t} \widetilde{S}(s) U(s ; \mu) d s, 0 \leq t \leq L,
$$

where $\tilde{S}(t)$ is given by (3.15) and $I_{2 n}$ is the $2 n \times 2 n$ identity matrix. Partition $U(t ; \mu)$ as a $2 \times 2$ block matrix according to the partitioning of the right hand side of equation (3.15) :

$$
U(t ; \mu) ;=\left[\begin{array}{ll}
U_{11}(t ; \mu) & U_{12}(t ; \mu) \\
U_{21}(t ; \mu) & U_{22}(t ; \mu)
\end{array}\right] .
$$

Then,

( i ) $\quad I_{2 n}-\mu \widetilde{P} \widetilde{Q}$ is invertible if only and if

$$
I_{2 n}-\left[\begin{array}{cc}
\mu P Q & \mu P \\
-U_{21}(L ; \mu) & I_{n}-U_{22}(L ; \mu)
\end{array}\right]
$$

is invertible.

(ii) $\lambda \neq 0$ is the eigenvalue of $\tilde{P} \tilde{Q}$ if and only if

$$
\begin{aligned}
& \operatorname{det}\left(\mu^{-1} U_{22}(L ; \mu) P^{-1}\left(I_{n}-\mu P Q\right)+U_{21}(L ; \mu)\right) \\
& =0 .
\end{aligned}
$$

Proof: To prove the theorem, we need the following lemmas 2 and 3.

Lemma $2^{8)}$ : Let $\tilde{S}(t)$ be $2 n \times 2 n$ matrix whose entries are Lebesgue integrable on $0 \leq t \leq L$. Then for $\mu \in \mathscr{R}$, there exists a unique continuous $2 n \times 2 n$ matrix function $U(t ; \mu)$ such that

$$
U(t ; \mu)=I_{2 n}+\mu \int_{0}^{t} \widetilde{S}(s) U(s ; \mu) d s, 0 \leq t \leq L .
$$

Futhermore, det $U(t ; \mu) \neq 0$, and

$$
\begin{aligned}
& U^{-1}(t ; \mu)=I_{2 n}-\mu \int_{0}^{t} U^{-1}(s ; \mu) \widetilde{S}(s) d s, \\
& 0 \leq t \leq L .
\end{aligned}
$$

Proof: See the reference ${ }^{8)}$.

Lemma $3^{8)}$ : Let $H$ be defined by (3.12). Then for 
$\mu \in \mathscr{R}$ and $\varphi \in L_{2}[0, L],(I-\mu H)$ is invertible and for 0 $\leq t \leq L$,

$$
\begin{aligned}
& \left((I-\mu H)^{-1} \varphi\right)(t) \\
& =\varphi(t)+\mu H_{1}(t) U(t ; \mu) \int_{0}^{t} U^{-1}(s ; \mu) \\
& \quad \times H_{2}(s) \varphi(s) d s,
\end{aligned}
$$

where

$$
H_{1}(t)=\left[\begin{array}{ll}
F_{1}(t) & F_{2}(t)
\end{array}\right], H_{2}(t) \doteq\left[\begin{array}{c}
G_{1}(t) \\
-G_{2}(t)
\end{array}\right],
$$

and, $F_{1}, F_{2}, G_{1}$ and $G_{2}$ are given by (3.10).

Proof: See the reference ${ }^{8}$.

Proof of theorem 2: (i ) From (3.14), $\left(I_{2 n}\right.$ $-\mu \tilde{P} \widetilde{Q})$ can be rewritten by

$$
\underbrace{\left[\begin{array}{cc}
I_{n} & 0 \\
0 & I_{n}-\mu H
\end{array}\right]}_{X} \underbrace{-\mu\left[\begin{array}{cc}
P Q & P \\
K_{1} & K_{2}
\end{array}\right]}_{Y} \underbrace{\left[\begin{array}{cc}
I_{n} & 0 \\
0 & K_{1}^{*}
\end{array}\right]}_{Z} .
$$

Recall that the first term term of (4.9) is an operator of Volterra type, the second term is a finite-rank matrix operator. Futhermore, since $X$ is an invertible operator, $X-Y Z$ is invertible if and only if ( $I$ $\left.-Z X^{-1} Y\right)$ is invertible. Thus, it follows that for $\mu \in$ $\mathscr{R},\left(I_{2 n}-\mu \widetilde{P} \tilde{Q}\right)$ is invertible if only and if the following matrix :

$$
I-Z X^{-1} Y=I_{2 n}-\mu\left[\begin{array}{cc}
P Q & P \\
K_{1}^{*} H_{\mu}^{-1} K_{1} & K_{1}^{*} H_{\mu}^{-1} K_{2}
\end{array}\right]
$$

is invertible, where $H_{\mu}=\left(I_{n}-\mu H\right)$. From lemmas 2 and $3,(3.8)$ and (3.9), (2.1) and (2.2) entries of the last right hand side of (4.10) are given by :

$$
\begin{aligned}
& \left(\left(I_{n}-\mu H\right)^{-1} K_{1} z\right)(t) \\
& =\left\{H_{1}(t)+\mu H_{1}(t) U(t ; \mu)\right. \\
& \left.\quad \times \int_{0}^{t} U^{-1}(s ; \mu) \widetilde{S}(s) d s\right\}\left[\begin{array}{l}
z \\
0
\end{array}\right] \\
& =\left\{H_{1}(t)+H_{1}(t) U(t ; \mu)\left(I_{2 n}-U^{-1}(t ; \mu)\right)\right\}\left[\begin{array}{l}
z \\
0
\end{array}\right] \\
& =H_{1}(t) U(t ; \mu)\left[\begin{array}{l}
z \\
0
\end{array}\right]
\end{aligned}
$$

It follows that

$$
\begin{aligned}
& K_{1}^{*}\left(I_{n}-\mu H\right)^{-1} K_{1} z \\
& \quad=\int_{0}^{L} G_{2}(s) H_{1}(s) U(s ; \mu)\left[\begin{array}{l}
z \\
0
\end{array}\right] d s \\
& \quad=\left[0-I_{n}\right] \int_{0}^{L} H_{2}(s) H_{1}(s) U(s ; \mu)\left[\begin{array}{l}
z \\
0
\end{array}\right] d s \\
& \quad=\left[0-I_{n}\right] \int_{0}^{L} \widetilde{S}(s) U(s ; \mu)\left[\begin{array}{l}
z \\
0
\end{array}\right] d s
\end{aligned}
$$

$$
\begin{aligned}
& =\mu^{-1}\left[0-I_{n}\right]\left(U(L ; \mu)-I_{2 n}\right)\left[\begin{array}{l}
z \\
0
\end{array}\right] d s \\
& =-\mu^{-1} U_{21}(L ; \mu) z .
\end{aligned}
$$

In a similiar way,

$$
\left(\left(I_{n}-\mu H\right)^{-1} K_{2} z\right)(t)=H_{1}(t) U(t ; \mu)\left[\begin{array}{l}
0 \\
z
\end{array}\right]
$$

It follows that

$$
K_{1}^{*}\left(I_{n}-\mu H\right)^{-1} K_{2} z=\mu^{-1}\left(I_{n}-U_{22}(L ; \mu)\right) z
$$

Therefore, it follows that (4.10) can be rewritten by

$$
I_{2 n}-Z X^{-1} Y=\left[\begin{array}{cc}
I_{n}-\mu P Q & -\mu P \\
U_{21}(L ; \mu) & U_{22}(L ; \mu)
\end{array}\right],
$$

and we proved the theorem 2 (i ) completely.

(ii) Take $\lambda \neq 0$. Then $\lambda$ is an eigenvalue of $\widetilde{P} \widetilde{Q}$ if and only if the finite rank matrix of (4.13) is not invertible. In other words, $\lambda$ can be computed by

$$
\begin{aligned}
\operatorname{det} & {\left[\begin{array}{cc}
I_{n}-\mu P Q & -\mu P \\
U_{21}(L ; \mu) & U_{22}(L ; \mu)
\end{array}\right] } \\
= & \operatorname{det}\left[\mu^{-1} U_{22}(L ; \mu) P^{-1}\left(I_{n}-\mu P Q\right)\right. \\
& \left.+U_{21}(L ; \mu)\right] \\
= & 0 .
\end{aligned}
$$

Hence we proved theorem 2 (ii).

Note that $\sigma_{i}(G)$ can be computed by the computation of the gramians, $P$ and $Q$ of $F(s)$, and the matrix function $U(L ; \mu)$ defined by $(4.1)$, where $U(t ; \mu)$ is called the fundamental matrix (normalized to $I_{2 n}$ at $t$ $=0$ ) of a homogeneous time varying differential equation.

Glover et al. ${ }^{6}$ computed the Hankel singular values of $G(s)$ from the following transcendental equation:

$$
\operatorname{det}\left(E_{1} e^{E L}+E_{2}\right)=0,
$$

where

$$
\begin{aligned}
& E=\left[\begin{array}{cc}
A & \sigma^{-1} B B^{*} \\
-\sigma^{-1} C^{*} C & -A^{*}
\end{array}\right], \\
& E_{1}=\left[\begin{array}{cc}
0 & 0 \\
\sigma^{-1} Q & -I_{n}
\end{array}\right], E_{2}=\left[\begin{array}{cc}
-I_{n} & \sigma^{-1} P \\
0 & 0
\end{array}\right],
\end{aligned}
$$

and $P, Q$ are the positive definite Hermitian solutions of (3.6), (3.7), respectively.

On the computation of the Hankel singular values, the theorem 2 is different from the result of (4.14) only in obtaining the fundamental solutions, $U(L ; \mu)$ and $\exp (E L)$. This difference is explained by the following theorem 3. explicitly.

Theorem 3 : Let $U(t ; \mu)$ and $\exp (E L)$ be defined by (4.1) and (4.15). Then, there exists a coordinate transformation matrix function $T_{i v}(t)$ such that

$$
T_{i v}(t) U(t ; \mu) T_{i v}^{-1}(0)=\exp (E t),
$$


where $T_{i v}(t)$ is given by

$$
T_{i v}(t)=\left[\begin{array}{cc}
e^{A t} & 0 \\
\frac{1}{\sigma} Q e^{A t} & \frac{1}{\sigma} e^{-A^{\prime} t}
\end{array}\right] .
$$

Proof : From a straightforward calculation, it is easily shown that there exists $T_{i v}$ of (4.17) such that

$$
T_{i v}(t)\left(\frac{1}{\sigma^{2}} \widetilde{S}(t)\right) T_{i v}^{-1}(t)+\frac{d}{d t} T_{i v}(t) T_{i v}^{-1}(t)=E .
$$

Thus, we have that two differential equations are connected through a coordinate transformation matrix $T_{i v}(t)$. Then, (4.16) is immediately derived by (4.18).

Note that it follows from (4.16) that the result of (4.4) is converted into that of (4.14), so is the converse.

\section{Perturbation Theory of the Hankel Singular Values}

In this section, using the minimax proprties of the eigenvalues of a compact and non-negative selfadjoint operator, we will obtain lower and upper bounds of the Hankel singular values.

\section{1 Minimax Theorem of the Eigenvalues}

Definition : Let $T$ be a linear bounded operator on Hilbert space $\mathscr{H}$. $T$ is called non-negative if $\langle T x, x\rangle$ $\geq 0$ for $x \in \mathscr{H}$.

Note that a compact self-adjoint operator $T$ is non-negative if and only if its eigenvalues are nonnegative.

Recall that all the eigenvalues of a compact and self-adjoint operator on Hilbert space $\mathscr{H}$ are real and countable set that has only zero as a limit point and eigenvectors corresponding to distinct eigenvalues are orthogonal. Then, the minimax theorem is a consequence of the spectral theorem.

Lemma 4[MiniMax Theorem ${ }^{12)}$ ]: Let $T$ be a compact and non-negative self-adjoint linear operator on Hilbert space, $\mathscr{H}$, and let $\lambda_{1} \geq \lambda_{2} \geq \cdots$, be the eigenvalues of $T$. Then for each positive integer $i$,

$$
\lambda_{i}(T)=\min _{\substack{S \\ \operatorname{dim} S=i-1}} \max _{\substack{\|x\|=1 \\ x \perp S}}\langle x, T x\rangle .
$$

Lemma 5: Let $T$ be a compact and non-negative self-adjoint operator mapping $\mathscr{H}$ into $\mathscr{H}$ and $\mathscr{H}_{1}$ be a subspace of $\mathscr{H}$. Suppose that there exists $T_{1}: \mathscr{H}_{1} \rightarrow$ $\mathscr{H}_{1}$ such that $T_{1}=\left.\Pi T\right|_{\mathscr{H}_{1}}$, where $\Pi$ is the orthogonal projection operator from $\mathscr{H}_{b}$ onto $\mathscr{H}_{1}$. Let $\lambda_{i}(T)$, $\lambda_{i}\left(T_{1}\right)$ be the eigenvalues of $T, T_{1}$, arranged in descending order, respectively. Then

$$
\lambda_{i}(T) \geq \lambda_{i}\left(T_{1}\right) \text {. }
$$

Proof: Let $\mathscr{H}_{1}, S$ be subspaces of Hilbert space $\mathscr{H}_{\mathfrak{C}}$ and $\Pi$ be an orthogonal projection operator from $\&$ onto $\mathscr{H}_{1}$. Then

$$
\Pi S=\mathscr{H}_{1} \cap\left(\mathscr{H}_{1}^{\perp} \cap S\right)^{\perp} .
$$

Let $\mathscr{H}_{0}$ be any $(i-1)$ dimensional subspace of $\mathscr{H}$. Then it follows from lemma 4 that

$$
\lambda_{i}(T)=\max _{\substack{\|x\|=1 \\ x \perp \mathscr{F}_{0}}}\langle x, T x\rangle,
$$

and from (5.4), $S_{0}=\mathscr{H}_{1} \cap\left(\mathscr{H}_{1}^{\perp} \cap \mathscr{H}_{0}\right)^{\perp}=\Pi \mathscr{H}_{0}, \operatorname{dim} S_{0}$ $\leq i-1$ is a subspace of $\mathscr{H}_{1}$. Then if we take an $(i-1)$ dimensional subspace $S_{1}$ of $\mathscr{H}$ such that $S_{0} \subset S_{1}$ of $\mathscr{H}$, it follows from lemme 4 that

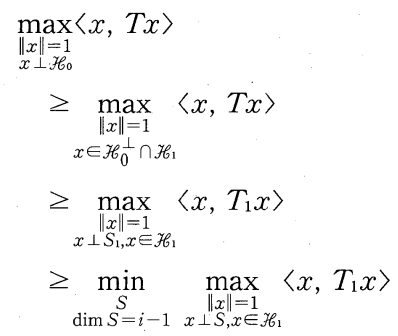

Thus we obtain $\lambda_{i}(T) \geq \lambda_{i}\left(T_{1}\right)$.

\section{2 Upper and Lower Bounds on the Hankel Singular Values}

Since $\widetilde{P}^{-1}$ exists, the eigenvalue problem of $\tilde{P} \tilde{Q}$ may be transformed into that of

$$
\widetilde{P}^{1 / 2} \tilde{Q} \tilde{P}^{1 / 2} z_{f}=\lambda z_{f}, x_{f}=\tilde{P}^{1 / 2} z_{f},
$$

where $\widetilde{P}^{1 / 2} \widetilde{Q} \widetilde{P}^{1 / 2}$ is a compact and non-negative selfadjoint operator and is described as sum of two compact and self-adjoint operators, $\Gamma_{F}$ and $\Gamma_{P}$, where

$$
\begin{aligned}
\Gamma_{F} & =\left[\begin{array}{cc}
P^{1 / 2} Q P^{1 / 2} & 0 \\
0 & 0
\end{array}\right], \\
\Gamma_{P} & =\left[\begin{array}{cc}
0 & P^{1 / 2} K_{1}^{*} \\
K_{1} P^{1 / 2} & K
\end{array}\right] .
\end{aligned}
$$

The eigenvalues of their operators are computed by the following theorem 4 , respectively.

Theorem 4 : Let $\lambda_{F}\left(\mu_{F}^{-1} \neq 0\right)$ and $\lambda_{P}\left(\mu_{P}^{-1} \neq 0\right)$ be the eigenvalues of $\Gamma_{F}, \Gamma_{P}$, respectively. For $\mu_{P} \in \mathscr{R}$ be the unique $2 n \times 2 n$ matrix function determined by

$$
U\left(t ; \mu_{P}\right)=I_{2 n}+\int_{0}^{t} \tilde{S}(r) U\left(r ; \mu_{P}\right) d r, 0 \leq t \leq L,
$$

where $\widetilde{S}(t)$ is given by (3.15). Then 
(i ) $\Gamma_{F}$ is an eigenvalue of $\Gamma_{F}$ of if and only if $\operatorname{det}\left[\mu_{F}^{-2}\left(I_{n}-\mu_{F} P Q\right)\right]=0$.

(ii ) $\mu_{P}(\neq 0)$ is an eigenvalue of $\Gamma_{P}$ of if and only if

$\operatorname{det}\left[\mu_{P}^{-1} U_{22}\left(L ; \mu_{P}\right) P^{-1}+U_{21}\left(L ; \mu_{P}\right)\right]=0 .(5.11)$

Proof : It is evident that $\lambda_{F}$ satisfies (5.10). The proof of (ii) is the same as theorem 2 .

Note that theorem 3 implies there is a transcendental equation involving the Hamiltonian matrix that is equivalent to (5.11).

Using the lemmas 4 and 5, we have upper and lower bounds.

Theorem 5: Let $\lambda_{i}\left(\Gamma_{G}\right), \lambda_{i}\left(\Gamma_{F}\right), i=1,2, \cdots$, be the eigenvalues of $\Gamma_{G}, \Gamma_{F}$ of (5.7) and (5.8), arranged in the descending order, respectively. Then

$$
\lambda_{i}\left(\Gamma_{F}\right) \leq \lambda_{i}\left(\Gamma_{G}\right) \leq \lambda_{i}\left(\Gamma_{F}\right)+\left\|\Gamma_{P}\right\| .
$$

Proof : Let $\lambda_{i}\left(\Gamma_{F}\right)=\lambda_{i}\left(P^{1 / 2} Q P^{1 / 2}\right), i=1,2, \cdots, n$, be the eigenvalues of $\Gamma_{F}$ arranged in descending order. Let $\Pi$ be the orthogonal projection operator projecting $\mathscr{R}^{n} \times \mathscr{L}_{2}(0, L)$ onto $\mathscr{R}^{n}$. Then $\left.\Pi \Gamma_{G}\right|_{\mathscr{R}^{n}}=P^{1 / 2} Q P^{1 / 2}$. Using this fact and lemma 5 , lower bounds are given. It follows from lemma 4 that

$$
\begin{aligned}
& \lambda_{i}\left(\Gamma_{G}\right)=\min _{\substack{S \\
\operatorname{dim} S=i-1}} \max _{\substack{\| \xi=1 \\
\xi \perp S}}\left\langle\xi, \Gamma_{G} \xi\right\rangle, \\
& =\min _{\substack{S \\
\operatorname{dim} S=i-1 \xi \|=1}} \max _{\substack{\| \notin \perp \\
\xi}}\left(\left\langle\xi, \Gamma_{F} \xi\right\rangle+\left\langle\xi, \Gamma_{P} \xi\right\rangle\right), \\
& \leq \min _{S}\left(\max _{\|\xi\|=1}\left\langle\xi, \Gamma_{F} \xi\right\rangle+\left\|\Gamma_{P}\right\|\right), \\
& =\min _{S} \max _{\|\xi\|=1}\left(\left\langle\xi, \Gamma_{F} \xi\right\rangle+\left\|\Gamma_{P}\right\|\right) \text {, } \\
& =\lambda_{i}\left(\Gamma_{F}\right)+\left\|\Gamma_{P}\right\|,
\end{aligned}
$$

where $\| \bullet$ denotes the operator norm.

Note that $\left\|\Gamma_{P}\right\|$ is bounded by the Hilbert-Schmidt norm, $\left\|\Gamma_{P}\right\|_{H . s .}$., i. e., $\left\|\Gamma_{P}\right\| \leq\left\|\Gamma_{P}\right\|_{H . s^{8}{ }^{8} \text {. }}$

Next we consider in case that the length of delay is $L^{\prime} \leq L$. Let define $\Gamma_{G}\left(L^{\prime}\right): \mathscr{R}^{n} \times \mathscr{L}_{2}\left(0, L^{\prime}\right) \rightarrow \mathscr{R}^{n} \times \mathscr{L}_{2}\left(0, L^{\prime}\right)$, $\Gamma_{G}(L): \mathscr{R}^{n} \times \mathscr{L}_{2}(0, L) \rightarrow \mathscr{R}^{n} \times \mathscr{L}_{2}(0, L)$. Then we have the following corollary.

Corollary 1: Let $\lambda_{i}\left(\Gamma_{G}\left(L^{\prime}\right)\right), \lambda_{i}\left(\Gamma_{G}(L)\right)$ be the eigenvalues of $\Gamma_{G}\left(L^{\prime}\right), \Gamma_{G}(L)$ arranged in descending order. Then

$$
\lambda_{i}\left(\Gamma_{G}\left(L^{\prime}\right)\right) \leq \lambda_{i}\left(\Gamma_{G}(L)\right)
$$

Proof : Let $\Pi$ be the orthogonal projection operator from $\mathscr{R}^{n} \times \mathscr{L}_{2}(0, L)$ onto $\mathscr{R}^{n} \times \mathscr{L}_{2}\left(0, L^{\prime}\right)$. Then, it is easily shown that

$$
\left.\Pi \Gamma_{G}(L)\right|_{\mathscr{R}^{n} \times \mathscr{L}_{2}\left(0, L^{\prime}\right)}=\Gamma_{G}\left(L^{\prime}\right) .
$$

Thus, (5.15) can be obtained from (5.16) and lemma 5.

Note that this result means that as delay increases, all the Hankel singular values have a monotone increasing property.

\section{Numerical Example}

We consider a simple example as follows :

$$
G(s)=\frac{1}{s+a} e^{-L s}, a \geq 0, L \geq 0
$$

A differential-difference equation of (6.1) is given by

$$
\begin{aligned}
& \dot{x}(t)=-a x(t)+u(t-L), \\
& y(t)=x(t),
\end{aligned}
$$

where $A=-a, B=C=1$ and $P=Q=1 / 2 a$. And semi-seperable kernel functions defined by (3.10) and $\widetilde{S}(t)$ of (3.15) are

$$
\begin{aligned}
& F_{1}(t)=G_{2}(t)=\frac{1}{2 a} e^{-a t}, F_{2}(t)=G_{1}(t)=e^{a t} . \\
& \widetilde{S}(t)=\left[\begin{array}{cc}
\frac{1}{2 a} & e^{2 a t} \\
-\frac{1}{4 a^{2}} e^{-2 a t} & -\frac{1}{2 a}
\end{array}\right],
\end{aligned}
$$

and the fundamental solution $U(t ; \mu)$ of (4.1) is given by a straightforward calculation of the differential equation : for $U(0 ; \mu)=I$,

$$
\frac{d}{d t} U(t ; \mu)=\mu\left[\begin{array}{cc}
\frac{1}{2 a} & e^{2 a t} \\
-\frac{1}{4 a^{2}} e^{-2 a t} & -\frac{1}{2 a}
\end{array}\right] U(t ; \mu) .
$$

The entries $U_{21}$ and $U_{22}$ of a fundamental solution $U(\mu ; t)$ defined by (4.1) are as follows:

$$
\begin{aligned}
U_{21}(t ; \mu)= & \frac{e^{-a t}}{4 a^{2}}\left[\frac{\mu}{2 \alpha}\left(e^{-\alpha t}-e^{\alpha t}\right)\right], \\
U_{22}(t ; \mu)= & e^{-a t}\left[\frac{1}{2}\left(e^{\alpha t}+e^{-\alpha t}\right)\right. \\
& \left.+\frac{a-\mu / 2 a}{2 \alpha}\left(e^{\alpha t}-e^{-\alpha t}\right)\right],
\end{aligned}
$$

where $\alpha^{2}=a^{2}-\mu$. Note that the choice of the square root is not essential here because the formulas do not change if $\alpha$ is replaced by $-\alpha$. Hence, formula (4.4) in theorem 2 means that the Hankel singular values $\sigma_{G}^{2}\left(=\mu_{G}^{-1}\right)$ are calculated by the following equation :

$$
\tanh \left(L \alpha_{G}\right)=-\frac{\alpha_{G}\left(3 a^{2}+\alpha_{G}{ }^{2}\right)}{a^{3}+3 a \alpha_{G}{ }^{2}},
$$

where $\alpha_{G}^{2}=a^{2}-\mu_{G}$. It is noted that the condition that a solution of (6.6) exists is $\mu_{G} \geq a^{2}$ and as $L$ increases or $a$ decreases, $\mu_{G}$ decreases ( $\sigma_{G}$ increases). Futhermore, $\lambda_{F}\left(=\mu_{F}^{-1}\right)$ of $\Gamma_{F}$ and $\lambda_{P}\left(=\mu^{-1}\right)$ of $\Gamma_{P}$ can be 
Table 1 Hankel singular values $(\sigma)$

\begin{tabular}{l|c|c|c}
\hline \hline & $L=0.5 \mathrm{sec}$ & $L=1.0 \mathrm{sec}$ & $L=1.5 \mathrm{sec}$ \\
\hline$\sigma_{1}$ & 0.6559 & 0.7373 & 0.7903 \\
$\sigma_{2}$ & 0.2186 & 0.3528 & 0.4545 \\
$\sigma_{3}$ & 0.0993 & 0.1863 & 0.2627 \\
$\sigma_{4}$ & 0.0621 & 0.1208 & 0.1761 \\
$\sigma_{5}$ & 0.0449 & 0.0885 & 0.1306 \\
$\sigma_{6}$ & 0.0351 & 0.0695 & 0.1033 \\
$\sigma_{7}$ & 0.0288 & 0.0572 & 0.0852 \\
$\sigma_{8}$ & 0.0244 & 0.0486 & 0.0725 \\
$\sigma_{9}$ & 0.0212 & 0.0422 & 0.0630 \\
$\sigma_{10}$ & 0.0187 & 0.0373 & 0.0557 \\
\hline
\end{tabular}

calculated by $(5.10),(5.11)$ in theorem 4 as follows:

$$
\begin{aligned}
& \operatorname{det}\left[\mu_{F}^{-1}\left(1-\frac{1}{4} \mu_{F}\right)\right]=0, \\
& \tanh \left(L \alpha_{P}\right)=\frac{16 a^{3} \alpha_{P}}{\alpha_{P}^{4}-10 a^{2} \alpha_{P}^{2}-7 a^{4}},
\end{aligned}
$$

where $\alpha_{P}^{2}=a^{2}-\mu_{P}$. Thus, $\lambda_{F}=\{1 / 2 a, 0,0, \cdots\},\left\|\Gamma_{P}\right\|$ is bounded by

$$
\begin{aligned}
\left\|\Gamma_{P}\right\|_{\text {H.S. }} & =2\left\|P^{1 / 2} K_{1}^{*}\right\|_{\text {H.S. }}+\|K\|_{\text {H.S. }} \\
& =\frac{L}{128 a^{4}}\left(33 L-16-L e^{-4 a L}-16 e^{-2 a L}\right) .
\end{aligned}
$$

Table 1 shows the Hankel singular values for $L=$ $0.5,1$ and $1.5 \mathrm{sec}$. The monotone increasing property is readily seen from the table.

\section{Conclusions}

In this paper, using the property that the gramian is described as an integral operator with semi-seperable kernel function, it was shown that the Hankel singular values of input time delay systems satisfy a transcendental equation. Futhermore, using minimax properties of compact an non-negative self adjoint operators, lower and upper bounds of the Hankel values were given. An implication of this is that as delay increases, all the Hankel singular values have a monotone increasing property.

\section{References}

1) K. Glover : All Optimal Hankel-norm Approximation of Linear Multivariable Systems and Their $\mathscr{L}^{\infty}$-error Bounds, Int. J. Control, 39-6, 1115/1193 (1984)

2) K. Glover, R. F. Curtain and J. R. Partington: Realisation and Approximation of Linear Infinite Dimensional Systems with Error Bounds, SIAM J. on Control and Optimization, 26-4, 863/898 (1988)

3) K. Glover, J. Lam and J.R. Partington: Rational Approximation of a Class of Infinite-Dimensional Systems II : Optimal Convergence Rates of $\mathscr{L}^{\omega}$ Approximants, Math. Control Signals Systems, 4, 233/246 (1991)

4) D. S. Flamm and S. K. Mitter: $\mathscr{H}^{\infty}$ Sensitivity Minim- ization Problem for Delay Systems, Systems \& Control Letters, 9, 17/24 (1987)

5) K. Zhou and P.P. Khargenekar: On the Weighted Sensitivity Minimization Ploblem for Delay Systems, Systems \& Control Letters, 8, 307/312 (1987)

6) K. Glover, J. Lam and J. R. Partington: Rational Approximation of a class of Infinite-Dimensional Approximation of a class of Infinite-Dimensional Systems I : Singular Values of Hankel Operators, Math. Control Signals Systems, 3-4, 325/344 (1990)

7) K. Kojima and S. Ishijima: On Balanced Reduction for Input Time Delay Systems, The 23-th Control Theory Symposium, 265/270 (1994)

8) I. Gohberg, S. Goldberg and M. A. Kaasheok: Classes of Linear Operators, Vol. I, Operator Theory : Advances and Applications, 49, Birkhäuser (1990)

9) B. A. Bamieh and J. B. Pearson: A General Framework for Linear Periodic Systems with Applications to $H^{\infty}$ Sampled-Data Control, IEEE Trans., AC $-37-4$, 418/435 (1992)

10) J. R. Partington: An Introduction to Hankel Operators, London Mathematical Society Student Texts 13, Cambridge University Press

11) R. F. Curtain: Robust Stabilizing of Normalized Coprime Factors: the Infinite-Dimensional Case, Int. .J. Control, 51-6, 1173/1190 (1990)

12) I. Goldberg and S. Goldberg: Basic Operator Theory, Birkhäuser (1981)

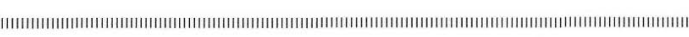
Authors' Profils

\section{Cheol Hwang (Student Member)}

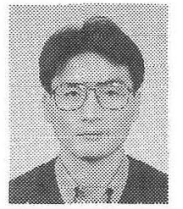

$\mathrm{He}$ received the B.E. and M.E. degrees in Mechanical Design Engineering from Pusan National University, Korea, in 1987 and 1991, respectively. From 1987 to 1988, he was engaged in Samsung Heavy Industry Co. in Korea. $\mathrm{He}$ is currently doctor course in Osaka University. His research interests are in model reduction and robust control theory for infinite-dimensional systems.

\section{Hirohisa Nishino}

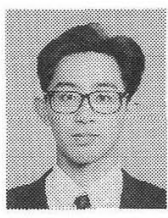

He received the B.E. and M.E. degrees in Mechanical Engineering for Computer-Controlled Machinery Faculty of Eng. from Osaka University, in 1993 and 1995, respectively. Currently, he is working in Mitsbishi Electric Co.. His research interests are in model reduction and robust control for infinitedimensional systems, in particular, acoustic systems. 
Yoshito OHтA (Member)

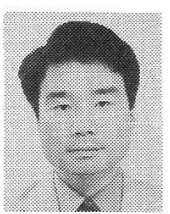

Yoshito Ohta received D. E. degree in electronic engineering from Osaka University in 1986. From 1983 to 1991, he was a Research Associate at the Department of Electronic Engineering, Osaka University. From 1986 to 1988, he was a Visiting Scientist at Laboratory for Information and Decision Systems, Massachusetts Institute of Technology. Since 1994, he has been an Associate Professor at the Department of Mechanical Engineering for ComputerControlled Machinery, Osaka University. His research interests includes robust control theory. 